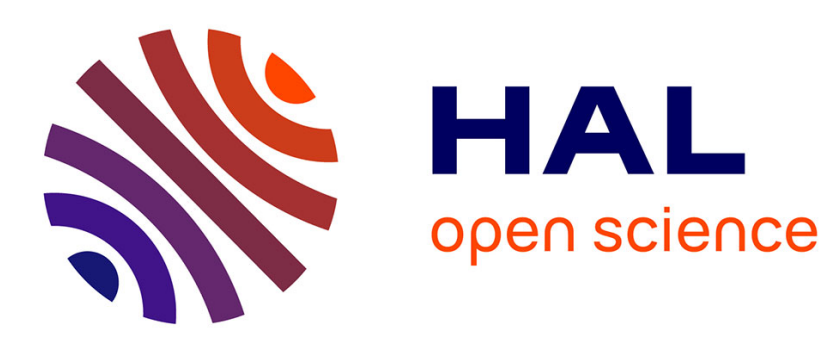

\title{
Making psychiatric semiology great again: A semiologic, not nosologic challenge
}

Jean-Arthur Micoulaud-Franchi, C. Quiles, Jean-Marie Batail, C. Lancon, M. Masson, Guillaume Dumas, M. Cermolacce

\section{- To cite this version:}

Jean-Arthur Micoulaud-Franchi, C. Quiles, Jean-Marie Batail, C. Lancon, M. Masson, et al.. Making psychiatric semiology great again: A semiologic, not nosologic challenge. L'Encéphale, 2018, 44 (4), pp.343-353. 10.1016/j.encep.2018.01.007 . hal-01920628

HAL Id: hal-01920628

https://hal-univ-rennes1.archives-ouvertes.fr/hal-01920628

Submitted on 13 Nov 2018

HAL is a multi-disciplinary open access archive for the deposit and dissemination of scientific research documents, whether they are published or not. The documents may come from teaching and research institutions in France or abroad, or from public or private research centers.
L'archive ouverte pluridisciplinaire HAL, est destinée au dépôt et à la diffusion de documents scientifiques de niveau recherche, publiés ou non, émanant des établissements d'enseignement et de recherche français ou étrangers, des laboratoires publics ou privés. 

6393 words
Making psychiatric semiology great again: a semiologic, not nosologic challenge

Perspective / Opinion

\section{Jean-Arthur MICOULAUD-FRANCHI ${ }^{1,2}{ }^{*}$, Clélia QUILES ${ }^{3,4}$, Jean-Marie BATAIL ${ }^{5,6}$,} Christophe LANCON ${ }^{7,8}$, Marc MASSON ${ }^{9,10}$, Guillaume DUMAS ${ }^{11,12,13,14}$, Michel CERMOLACCE ${ }^{7,15}$

1 - Services d'explorations fonctionnelles du système nerveux, Clinique du sommeil, CHU de Bordeaux, Place Amélie Raba-Leon, 33076 Bordeaux, France.

2 - USR CNRS 3413 SANPSY, CHU Pellegrin, Université de Bordeaux, France.

3 - Centre Hospitalier Charles Perrens, Pôle Universitaire de Psychiatrie Adulte, 121 Rue de la Béchade, 33076 Bordeaux Cedex, France

4 - Université Bordeaux Segalen, 146 rue Léo-Saignat, 33076 Bordeaux Cedex, France

5 - Academic Psychiatry Department, Centre Hospitalier Guillaume Régnier, Rennes, France 6 - EA 4712 Behavior and Basal Ganglia, CHU Rennes, Rennes 1 University, France.

7 - Pôle de Psychiatrie "Solaris", Centre Hospitalier Universitaire de Sainte-Marguerite, 270 Bd de SainteMarguerite, 13009 Marseille, France.

8 - Laboratoire de santé publique évaluation des systèmes de soins et santé perçue, Université de la Méditerranée - EA 3279 - Faculté de Médecine, 27 bd Jean Moulin, 13385 Marseille cedex 05, France.

9 - Nightingale Hospitals Paris-Clinique du château de Garches, France

10 - Service hospitalo-universitaire, Centre Hospitalier Sainte-Anne, 75015 Paris, France

11 - Institut Pasteur, Human Genetics and Cognitive Functions Unit, Paris, France

12 - CNRS UMR 3571 Genes, Synapses and Cognition, Institut Pasteur, Paris, France

13 - University Paris Diderot, Sorbonne Paris Cité, Paris, France

14 - Centre de Bioinformatique, Biostatistique et Biologie Intégrative, Paris, France

15 - Laboratoire de Neurosciences Cognitives (LNC), UMR CNRS 7291, 31 Aix-Marseille Université, Site St Charles, 3 place Victor Hugo, 13331 Marseille cedex 3, France

* Corresponding author:

Dr. MICOULAUD FRANCHI Jean-Arthur

Services d'explorations fonctionnelles du système nerveux, Clinique du sommeil, CHU de Bordeaux, Place Amélie Raba-Leon, 33076 Bordeaux 


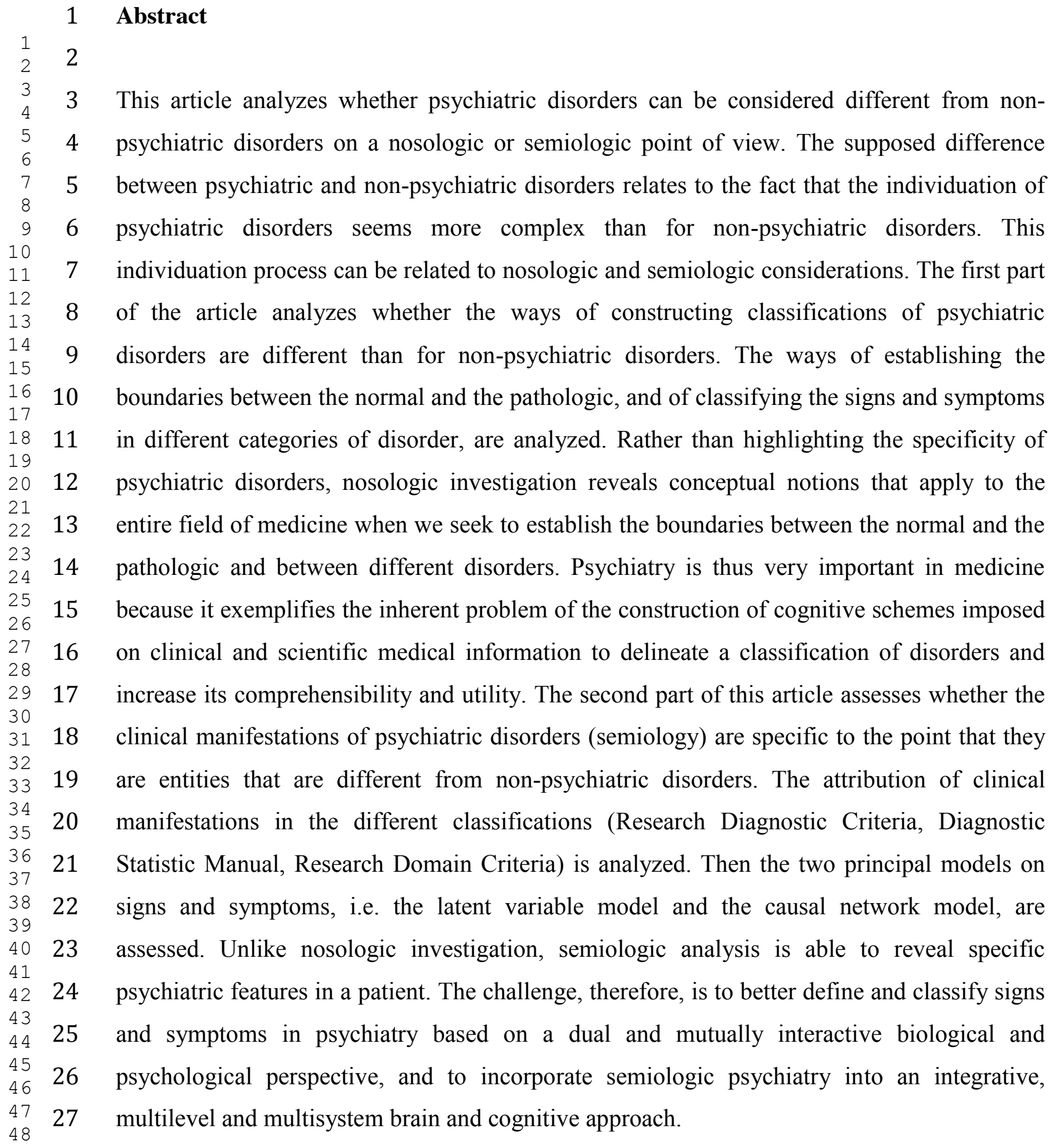




\section{Introduction}

4

5 Are psychiatric disorders different when considered from a nosologic point of view?

Evolution of nosologic issues from the RDC to the RDoC

Boundaries between the normal and the pathologic

Classification of different disorders: boundaries between disorders

9

Are psychiatric disorders different when considered from a semiologic point of view?

Evolution of semiologic thinking from the RDC to the RDoC

Psychiatric semiology as seen by the latent variable model

Psychiatric semiology as seen by the causal network model 


\section{Introduction}

2 A passionate long-lasting debate in medicine is knowing "what kinds of things are psychiatric 3 disorders?" [1]. This question has engendered complex historical, sociological, 4 epistemological and philosophical concepts with large differences between models and schools of psychiatric thought [1-7]. Such thinking is essential for the development of psychiatric medicine but commonly gives the implicit impression that psychiatric disorders

\footnotetext{
1 The term semiology is used in this article as a synonym of the term symptomatology, presentation, manifestation or phenomenology of the disorder. In this article, we use the term semiology, in line with the French medical tradition from the early 19th century. In the English tradition, the term semiology has referred since the middle of the 17th century to the science of language. Thus, the English medical tradition is to use "symptomatology", "clinical presentation, manifestation or phenomenology". However, it should also be noted that the term phenomenology used in this sense can be considered as a misuse in clinical psychiatry. Indeed, current usage as a set of signs and symptoms of a patient with a psychiatric disorder is different from the original meaning, which concerned comprehending a patient's subjective self-experience (in line with the continental understanding of phenomenological philosophy).
} 


\section{Are psychiatric disorders different when considered from a nosologic point of view?}

2 The long-standing discussion accompanying the construction of contemporary classifications 3 of psychiatric disorders, in particular the Diagnostic and Statistical Manual of Mental 4 Disorders (DSM) [12], demonstrates that unlike in other medical disciplines, it is not easy to classify disorders in psychiatry, thereby reinforcing the impression that psychiatric disorders are something different. Psychiatric disorders might be entities different from non-psychiatric disorders because the ways of establishing the boundaries between the normal and the pathologic, and of classifying the signs and symptoms in different categories of disorder, are not the same. The first part of the article thus analyzes whether the ways of constructing classifications of psychiatric disorders are different than for non-psychiatric disorders.

\section{Evolution of nosologic issues from the RDC to the RDoC}

The ways of establishing the boundaries between the normal and the pathologic and the classification of the different disorders have to be evaluated for their reliability and their validity [12].

- Reliability (also called "precision") refers to reducing disagreement among clinicians about whether some phenomena are to be considered as pathologic or not and about the psychiatric diagnosis.

- Validity (also called "accuracy") is "the degree to which diagnostic criteria reflect the comprehensive manifestation of an underlying psychopathological disorder" [13].

The early contemporary classification, and in particular the Research Diagnostic Criteria (RDC), sought to establish diagnostic criteria in order to enhance reliability in psychiatry [14]. The RDC project was developed at the Washington University School of Medicine in St Louis. The criteria of the St Louis group are also known as the "Feighner criteria", because Feighner was the author of the seminal article summarizing criteria for 15 psychiatric conditions [15]. The development of the RDC project led to the major revisions in DSM-III [16]. It stated: "Since in DSM-I, DSM-II, and ICD-9 explicit criteria are not provided, the clinician is largely on his or her own in defining the content and boundaries of the diagnostic categories. In contrast, DSM-III provides specific diagnostic criteria as guides for making each diagnosis since such criteria enhance interjudge diagnostic reliability" [17]. The expectation was that each psychiatric disorder would be validated by its separation from other disorders. However, the validity of the RDC and the subsequent DSM has been largely criticized [18, 19]. Thus, the Research Domain Criteria (RDoC) was the most important 
1 classifying mental disorders based on dimensions of observable behavioral and 2 neurobiological measures" [20]. The RDoC, which interestingly used an acronym very close 3 to the RDC project, was developed at the National Institute of Mental Health (NIMH), an 4 agency of the United States Department of Health and Human Services and the largest 5 research organization in the world specializing in mental illness. Thomas Insel, who led the NIMH from 2002 until 2015, largely supported the RDoC project. By proposing a dimensional approach based on brain function, it was thought that the future nosology of psychiatric disorders would be more valid, i.e. closer to underlying physiopathological mechanisms [18].

Boundaries between the normal and the pathologic

In this section we analyze ways of establishing the boundaries between the normal and the 1 25 pathologic. The construction of contemporary classifications of psychiatric disorders requires operational criteria to reduce disagreement among clinicians regarding whether a phenomenon are pathologic or not (reliability) and to ensure that it is determined by an underlying impaired physiological mechanism (validity). In this paper, we term "inclusion criteria" the defining criteria used to address reliability and to identify signs and symptoms that may be considered as "clinically significant"; and "exclusion criteria" the defining criteria used to address validity and to identify signs and symptoms that are not to be considered as pathologic. How specific criteria are designed to position the boundaries between two disorders will be analyzed in the following section.

In the RDC, these additional criteria were not explicitly defined. However, Feighner et al. stated that "the first step is to describe the clinical picture of the disorder. This may be a single striking clinical feature or a combination of clinical features thought to be associated with one another. Race, sex, age at onset, precipitating factors, and other items may be used to define the clinical picture more precisely. The clinical picture thus does not include only symptoms" [15]. Thus, they clearly indicated that the boundary between the normal and the pathologic should to be determined by features other than only the signs and symptoms. In 1978, Spitzer and Endicott proposed a detailed list of operational criteria [21] that are shown in Table 1. They are the most detailed operational criteria known for fixing the boundaries between the normal and the pathologic. These criteria were the bases of the reduced criteria published in the subsequent DSM. The criteria of the different editions of the DSM are shown in Table 2. 
1 The "inclusion criteria" to identify signs and symptoms as "clinically significant" are criteria based on the consequence of the disorder: disability. The DSM-5 specifically discussed the consequence of such a position in the field of medical nosology (p 21.) [13]. Indeed, the classifications developed by the World Health Organization (WHO) have clearly differentiated the concepts of disorder (with the International Classification of Diseases ICD) and disability (with the International Classification of Functioning, Disability and Health - ICF). Disability refers to the consequence of the disorder and not to its definition. In psychiatric classification, disability is part of the boundary of the disorder. This is an

important difference between psychiatric and non-psychiatric disorders, and it is widely held that the boundary of a disorder in medicine is based on a biomarker that reflects its pathophysiological mechanism. However, this is to forget, as Claude Bernard pointed out, that boundaries between the normal and the pathologic cannot be defined primarily on the basis of physiology [9]. A boundary is to be set first on clinical and epidemiological factors pertaining to disability or future disability if no therapy is begun [22]. Biomarkers are the second step and are taken to reflect a disease process explaining the disability or the evolution toward disability. The Spitzer and Endicott 1978 criteria already highlighted this point by using "a condition in the fully developed or extreme form" because "in medicine many conditions are recognizable in an early form, frequently with the aid of laboratory tests, before they have any undesirable consequences" [21].

Interestingly, these two steps are considered in reverse order by many physicians, who consider that the boundaries between the normal and the pathologic are defined primarily from the point of view of the pathophysiology and biomarker. They do not consider disability as being entirely part of the definition of the disorder. The difference between a psychiatric and non-psychiatric disorder is that the consideration of disability remains directly and explicitly within the realm of psychiatry, whereas this notion may be forgotten because biomarkers exist for non-psychiatric disorders. "Unfortunately, in psychiatry we rarely have laboratory procedures which permit us to diagnose mental disorders before they have clinical manifestations" [21]. Fundamentally, however, the process of differentiating the normal and the pathologic remains the same for psychiatric and non-psychiatric disorders.

Nevertheless, in line with Claude Bernard who stressed that the physiological mechanisms underlying a pathology should be described in order for medicine to be considered as scientific, a challenge in psychiatry is to identify biomarkers in order to better evaluate disability and future disability, a process also called clinical staging. "To test the concept,

34 there is a clear need to develop illness markers that (...) have the capacity to track the extent 
1 of the disease process" [23]. Biomarkers will also be useful for probing the efficiency of 2 treatments [24] and identifying resilient subjects who could have a biomarker related to a 3 disorder but which does not appear to be affected by the disorder [25].

4 As biomarkers have not been developed in psychiatry, exclusion criteria have been 5 determined to identify signs and symptoms that are not considered to be due to an underlying 6 physiological deterioration, and thus which are not considered to be pathologic. Table 2. 7 Indeed, "the locus of the disturbance in a medical disorder is at least partly within the 8 organism" [21]. "This does not mean that factors outside of the organism are of no 9 importance" but that "largely within the organism with regard to either initiating or 10 maintaining the condition" [21]. The DSM criteria seem to be rather imprecise and not very 11 operational, because they are based on general sociological considerations about the 12 relationship between the individual and society. On the other hand, the Spitzer and Endicott 13 criteria determined three criteria to establish whether signs and symptoms can be attributed to 14 a supposed mechanism within the organism (criteria B1, B2 and C, in Table 1).

15 The use of such criteria is an important difference between psychiatric and non-psychiatric 16 disorders. In fact, the absence of a biomarker should not mask the fact that the identification of a physiological impairment underlying a disorder is an ideal in medicine that is not absolutely necessary. This ideal was called "the hard medical model" by Kendler, who stated that "the hard medical model, while historically critical in the evolution of disease conceptualization, is in fact an idealization which fully applies to only a small group of Mendelian, nutritional, infectious and parasitic disorders" [26]. Moreover, following on from Spitzer and Endicott who stressed that "the nature of what is wrong may be known or unknown, and may or may not be conceptualized in somatic or physiological terms", the MEsH defined disease as "a definite pathologic process with a characteristic set of signs and symptoms" and added that "its etiology, pathology, and prognosis may be known or unknown". The very important point here is that the pathophysiological mechanism may be unknown so it is important to conduct scientific research to identify these mechanisms, not necessarily to have them for conducting clinical medicine [27]. Indeed, medicine is also based on clinical science $[28,29]$. Nevertheless, the wide field of scientific psychiatric research concerning the investigation of what is wrong inside the brain, and more generally the organism, of patients with a psychiatric disorder places psychiatry entirely and rightfully within the realm of scientific medicine. 
Classification of different disorders: boundaries between disorders

3 In this section we analyze how different disorders are classified. The construction of 4 contemporary classifications of psychiatric disorders has led to operational criteria to reduce disagreement among clinicians about psychiatric diagnosis (reliability) and to ensure that the phenomenon is determined by an underlying impaired physiological mechanism (validity). Reliability has been addressed by defining an appropriate list of criteria to identify signs and symptoms that are easily recognized by a clinician and are clustered together. Validity has been addressed by defining criteria that ensure the possibility of different underlying impaired physiological mechanisms for different disorders. These validity criteria were primarily identified by Robin and Gruze in 1970 [30], then added to by Spitzer and Endicott in 1978 [21], by Kendler in 1990 [31] and by the DSM-5 in 2013 [13]. Table 3. established. This is an important difference between psychiatric and non-psychiatric disorder classifications. Indeed, it is widely considered that the classifications of disorders in medicine are based on biomarkers that reflect pathophysiological mechanisms. However, classifying disorders is a very complex process involving epidemiological, semiologic, prognostic, therapeutic, economic, and genetic factors [27, 32, 33]. Figure 1. As posited by Spitzer and Endicott in 1978, "the operation by which syndromes and disease are distinguished from each other and from symptoms consist of examining the extent to which the external correlated of interest differ. The more they differ, the more likely it is that the condition represents syndrome or disease and not mere symptoms" [21]. This classification of disorders is in line with the coherence theory of truth that postulates that "the best diagnoses we have are the ones that are heavily intertwined with other things we know about; that is, are 'well validated'. For individuals assigned to that class, we follow the strings and see all the other things that we learn about them" [34]. This paradigm involves the "examination of the entire medical classification system reveals that no single principle accounts for the allocation of disorders to various subgroupings" [21]. Of course, all these relations of validation should be integrated in a unified pathophysiological process in which a biomarker is solely the reflection, but not the primarily cause, of the classification. Moreover, in many cases, such biomarkers are in fact a concept resulting from many causes and mechanisms, i.e. " $a$ therapeutic target rather than an etiological node; that is, not something to find that would explain everything, but something to act upon that would dissolve everything" [35]. 
1 The difference between psychiatric and non-psychiatric disorders is that the validators are by and large explicit in psychiatric classifications and we do not have any reliable simple validator for psychiatric diagnoses, whereas they may be forgotten or considered as biomarkers in non-psychiatric disorders. Fundamentally, however, the process of classifying any type of disorder is the same and has to take into account the multifactorial and etiologically complex nature of the explanatory models in medicine [33]. Biological reductionism is less fruitful than explanatory pluralism in most psychiatric and nonpsychiatric disorders [32]. One may consider that the DSM-5 implicitly takes explanatory pluralism into account by stating that... "the boundaries between many disorder "categories" are more fluid over the life course than DSM-IV recognized, and many symptoms assigned to a single disorder may occur, at varying levels of severity, in many other disorders. These findings mean that DSM, like other medical disease classifications, should accommodate ways to introduce dimensional approaches to mental disorders, including dimensions that cut across current categories" [13]. The question raised by the DSM-5, in line with the RDoC project is whether the actual classification based on categories is the best way to describe the multifactorial and etiologically complex pathophysiological mechanisms implicated in psychiatric disorders. The DSM-5 position underlines the "real paradigm shift" that was proposed by the $\mathrm{RDoC}$ project. The $\mathrm{RDoC}$ paradigm shifts the heuristic from coherence at the theoretical level to support for experimental observation at the physiological level [36], in line with the traditional translational research approach [37]. Indeed, " the RDoC does not take as starting point the traditional view of disorders as symptoms complexes based largely on clinical descriptions" for which validators are required [20]. In contrast, the RDoC considered validity from the point of view of physiological and not clinical dysfunction. Thus, it did not categorize clinical signs and symptoms but proposed a dimensional framework in which the different functions of the brain are considered, and supposed that any of these dimensions can be impaired. However, this dimensional approach raises an important question regarding the link between the physiological and medical approach to a disorder, an issue related to the notion of continuity. Indeed, where do we situate the threshold for considering a brain function as pathologic when we also consider each dimension as being continuous? As discussed previously and summarized by Stein in a comment on the RDoC: "we need to accept that diagnosis system cannot "carve nature at her joints". Rather facts and value need to be continually re-assessed, to try optimize classification" [33].

34 disorders at the nosologic level. We have shown that the lack of biomarkers in psychiatry has 
1 led the psychiatric community to clearly and thoughtfully explore the inherent process of 2 establishing the boundaries between the normal and the pathologic and between the different 3 disorders in medicine. This process is as complex in psychiatry as in any other medical field. 4 This does not mean, however, that epistemology of psychiatry ought to be general as part of 5 epistemology in medicine, without the need for regional epistemology $[4,38]$. The historical 6 and sociological specificity of psychiatry should not be underestimated. Nevertheless, we 7 believe that the need for a regional epistemology is more semiologic than nosologic. Thus, the 8 question to be asked now is whether psychiatric disorders differ from non-psychiatric 9 disorders at the semiologic level, i.e. with regard to their clinical manifestations. 


\section{Are psychiatric disorders different when considered from a semiologic point of view?}

2 The discussions concerning the drafting of the DSM-III surprisingly involved little discussion 3 on semiologic issues in psychiatry. Nevertheless, as stated in the DSM-III, the separation 4 between "physical" (non-psychiatric) and "mental" (psychiatric) disorders is not based on pathophysiological mechanisms (organic versus psychologic) but "on the tradition of separating those disorders whose manifestations are primarily behavioral or psychological

$$
\text { (i.e., mental disorders) from those whose manifestations are not." Identifying psychiatric }
$$
manifestations was thus a central issue for the authors of the DSM-III in identifying what kind of entity psychiatric disorders really are. Mental manifestations (symptoms and signs) are considered as the "unit of analysis" that has to be captured in order to make a psychiatric diagnosis [38]. However, thinking about what a sign or symptom in psychiatry is has not kept in step with reflection on the nosologic aspects of psychiatry [38, 39]. Thus, the second part of this article analyzes whether certain manifestations of psychiatric disorders make them stand apart from non-psychiatric disorders.

\section{Evolution of semiologic thinking from the RDC to the RDoC}

Symptoms refer to the subject's that they experience and notice, while signs refer to manifestations that they may experience but which are also externally observable by others [38]. Whatever the type of psychiatric interview [39], the first essential step when identifying psychiatric symptoms and signs is to conduct a clinical reasoning process about the psychiatric disorder and to take the necessary clinical decisions [40]. In line with this medical position, DSM-III discussions focused on operationalizing the criteria in order to enhance the reliability of assessing psychiatric disorders and thus of the diagnosis [41]. The aim was not to reduce clinical psychiatry to a list of criteria but to consider that: "if the medical practitioner confines himself to diagnosis and to treatment based specifically on [diagnostic tools], the help he can give (...) is in general severely restricted. (...) However, if the doctor cannot make a useful diagnosis, he severely restricts his competence in a different way and 28 becomes immeasurably less useful to his patient" [40]. The RDC was developed with this in 29 mind, the first step in scientific medicine being the clinical examination of patients. In the 30 non-psychiatric fields of medicine, the pride of place accorded to non-clinical results obtained 31 from laboratory tests, imaging, etc. can lead the clinician to ignore the importance of the 32 clinical examination in the development of medicine. However, "to neglect this stage would 33 be to misunderstand the nature of scientific enquiry" [40]. "Our present sophisticated medical 34 knowledge has accrued because of centuries of observation and description, in which the 
1 describers and classifiers have played as dynamic and creative part as those concerned with 2 process" [40]. "No disease theory can be elaborated before the clinical syndrome has been recognized and labeled", "the value of those first step should not be underestimated" and "the second step can then be taken, which is to test the validity of various explanatory theories". Figure 2. The RDC project and the resulting DSM criteria were developed in order to enhance the value of the first step. Unfortunately, the list of criteria in the DSM became the reference for semiologic terms in psychiatry, thereby reducing and simplifying the corpus of signs and symptoms [42, 43]. Moreover, the organization of the corpus of signs and symptoms was based on nosologic categories and not on supposed underlying pathophysiological mechanisms. "In many cases, there are also observable bodily abnormalities [physiological abnormalities]; most evident in the case of anxiety states (...), but nowhere sufficient in themselves for a reliable classification" [40]. As a result, it was posited that among the many possible techniques for classifying psychiatric symptoms and signs, the easiest to implement was the clinical diagnosis [40].

This subordination of semiology to nosology may be of value given the historical aspects of signs and symptoms in psychiatry. Wakefield has called this the "conceptual validity" of signs and symptoms. Conceptual validity has allowed the psychiatric community to "delineate the domain of psychological condition that fall under the concept of disorder" [44]. Indeed, present-day semiology bears close links to nosographic thinking, which itself can be traced back to historical and "psychosocial" views of the individual. In other words: "Since doctors have been making examinations of 'mental state' from time immemorial, they do not have to start afresh each time but can utilize the experience of their predecessors" [40]. Thus, rather than semiologic analysis being used as the first step when testing disease theory, it has come to occupy a subservient role to the categorization of psychiatric disorders. Figure 2. This role of subservience also means that the relationship between semiology and nosography tends to be inversed. In medicine, it is traditionally postulated that signs and symptoms influence which disorder category is to be employed, but not that the disorder category will influence the types of symptoms and signs that are sought in a patient. In psychiatry, symptoms and signs tend to be influenced by the disorder category [45]. This also means that symptoms and signs in psychiatry tend to contain de facto the boundaries between the normal and the pathologic, and the classification of disorder. In some way, the two-step process in which medicine has developed, i.e. clinical description then neurophysiological theory of disorder, is biased on in that clinical descriptions are largely influenced by concepts of disorder category [46]. As Berrios stated "the DSM-III two-stage model, according to 
1 which symptom and disease recognition are independent cognitive events, did not work. (...)

2 The disease hypothesis absolutely controls the way mental symptoms are captured and 3 recognized" [47]. Figure 2.

4 One can even consider that the $\mathrm{RDoC}$ project turned the initial project of establishing a 5 classification of psychiatric symptoms upside down [40]. It posited that the first step is not to 6 establish clinical descriptions but to gain neurophysiological knowledge about cognitive and 7 brain mechanisms [18]. By doing so, it is propounds the opposite of what Wing, Cooper and 8 Sartorius say [40]. Figure 2. Thus, by turning the two-step process around, the RDoC may be seen as an attempt to reorganize the classification of psychiatric symptoms and signs on the basis of the underlying physiological mechanisms and not on the basis of the historical classification of disorders. Aragona clearly highlighted the fact that the RDoC turns the original project upside down [12]. "Both the neo-Kraepelinian DSM-III and the DSM-5 are based on the same assumption about validity: i.e., the provisional phenomenal descriptions (the individual mental disorders or, as in the DSM-5, the larger grouping of adjacent disorders) will be fully validated when some specific neurobiological factors are "discovered" that confirm that the phenomenal description really corresponds to a neurobiological disease. (...) The RDoC project, which is considered the most promising etiopathogenic approach at play in current debates, shares with the other etiopathogenic approaches a revolutionary shift. While in the neo-Kraepelinian approach validation research is expected to proceed from phenomenally defined disorders back to the discovery of their etiology, in the etiopathogenic approaches the direction is expected to be from "subpersonal" dysfunctions (of genes, brain processes, or cognitive mechanisms) ahead to the resulting phenomenal picture. (...) They all see mental pathologies as biomedical entities resulting form a dysfunction of physiological processes; hence, the difference is just in the direction of the discovery enterprise, i.e., from the syndrome to the underlying pathophysiology in the DSMs, from pathophysiology to the resulting syndromes in the etiopathogenic approaches." [12].

The need for a rethink was also highlighted by Maj's editorial in World Psychiatry [48] in relation with the article by Cuthbert [18]. Maj stated that "the gap between the proposed dimensions and the signs and symptoms that, as Cuthbert acknowledges, are "the actual clinical phenomena that bring patients to the clinic" is sensible in several areas. If the problem with the DSM categories may be that they are too distant from the level of neuroscience, the problem with at least some of the RDoC constructs may be that they are somewhat distant from the level of clinical phenomena" [48]. In fact, Cuthbert in his article 34 did not avoid this issue. He stressed that "Another issue concerns the relationship of the 
1 various RDoC measures to presenting signs and symptoms, since of course the latter are the 2 actual clinical phenomena that bring patients to the clinic. Establishing mechanistic relationships by which disruptions in the functioning of one or more constructs (as assessed

4 by various Units of Analysis) result in specified symptoms or impairments is considered as a central task for the RDoC project, and a major component of the grant funding program. Notwithstanding the translational research approach, the RDoC project is very much directed toward an understanding of the impairments that patients experience in their lives, and this desideratum was emphasized by the RDoC workgroup in nominating constructs" [18]. Thus, the $\mathrm{RDoC}$ project may be seen more as a semiologic project than a nosologic one. The paradigm shift between the RDC and the RDoC is thus a reversal of the link between clinical descriptions of and scientific knowledge about disorders with a view to driving scientific psychiatric medicine forward. Nevertheless, the problem remains as to what signs and symptoms are in psychiatry, and whether semiologic in psychiatry may be considered as an important difference between psychiatric and non-psychiatric disorders. In fact, the theoretical foundations of how semiology was to be incorporated in the DSM-III received very little attention at the time of its conceptualization, and tended to be discussed only subsequently $[42,49]$.

The following section thus describes how signs and symptoms are to be considered in psychiatry. It has been stressed that: "What the patient manifests is not an isolated series of independent referring symptoms/signs but rather certain wholes of interpenetrating experiences, feelings, expressions, beliefs, and actions, all permeated by biographical detail. These aspects and these wholes are not constituted by a reference to underlying substrate (...) but by their meaning [50]". Thus, an important question concerning mental manifestations is their coherence. Coherence can be analyzed from an external point of view, which is called explanation or "erklären" by Jaspers. That is: what is the explanation and the mechanism that link together a set of clinical manifestations? The response is here driven by a putative causal substrate. Coherence can also be analyzed from an internal point of view, which is called understanding or "verstehen" by Jaspers [38]. That is: what is the meaning of a set of symptoms for the subject him/herself? The response is driven here by the comprehensive understanding of the individual rather that the symptoms and signs. Indeed, mental manifestations are clearly part of the entire self-experience of the subject, associated with biographical detail and having a personal meaning. This subjective aspect of signs and symptoms has been largely described by phenomenology, for a review, see: $[10,11,39,42$, 
1 points of view are presented: the so-called latent variable model, i.e. the traditional medical 2 one, which considers them as the manifestation of a given underlying pathophysiological mechanism; and the so-called causal network model, i.e. a more psychiatric-specific point of view in which signs and symptoms have their own causal relationship.

\section{Psychiatric semiology as seen by the latent variable model}

Symptoms and signs in non-psychiatric medicine are traditionally considered as an indicator of a latent variable [35]. A latent variable is a variable that is not directly observed but rather inferred from other variables that are observed. This latent variable is taken to be related to a physiological mechanism and thus to be the cause of the semiologic manifestation. Signs and symptoms are considered as independent things - independent from other signs and symptoms and from the context - but are considered to be dependent with regard to the causal physiological substrate that is to be identified.

Two levels of causal physiological substrate and latent variable may be identified, one at the semiologic level and the other at the nosologic level. Signs and symptoms are traditionally considered as manifestations that may be associated with many different disorders. Nevertheless, in non-psychiatric medicine each sign and symptom can be associated with a latent variable that is not a physiological mechanism of the disorder but of the signs and symptoms themselves. We propose to call this latent variable the "distal" latent variable. A distal latent variable may be explained by a different disorder that we propose to refer to as the "proximal" latent variable. The proximal latent variable should explain the occurrence of symptom and signs in a defined disorder, the course of the disorder, its comorbidity, and the treatment response. This distinction between the distal and the proximal is to be found in many fields of medicine. Non-psychiatric semiology has been largely organized until now on the basis of the distal aspects of physiology. The distal is important in any attempt to organize semiologic terminology independently of nosographic issues. To our knowledge, the classification task force of the International League Against Epilepsy (ILAE) is the group that has proposed the best differentiation of the semiologic [52] and the nosographic [53] .

The fact that signs and symptoms in psychiatry have not been organized in physiological terms based on the identification of a distal latent variable may be considered as an important difference between psychiatric and non-psychiatric entities. The cause of this putative difference needs to be analyzed thoroughly.

First, for non-psychiatric disorders, a semiologic organization based on neurophysiological factors is possible because of the consensus regarding the framework for classifying 
1 physiological functions. Although the framework is probably not complete, it is sufficient for 2 organizing signs and symptoms on a practical basis. Despite Cuthbert's statement that "the majors systems of the brain have been delineated and related to their functional outputs" 4 [18], psychiatric semiology may be considered as lacking such a consensual neurophysiological function framework [54]. Thus, symptoms and signs are organized rather according to clinical considerations than physiological ones.

Second, the signs and symptoms of non-psychiatric disorders can generally be considered as “objects", i.e. things that are "publicly accessible and involving mutually independent (and typically atomic) entities devoid of subtle or complex forms of meaning, and suitable for context-independent definition and measurement" [39]. In psychiatric semiology, it is more difficult to describe signs and symptoms solely as "objects". As Spitzer and Endicott stated in 1978, signs and symptoms are "primarily" psychological in nature [21]. This does not mean that signs and symptoms in non-psychiatric disorders are not subjective. Indeed, these aspects have already been studied in the field of health psychology [55]. However, it does mean that signs and symptoms in psychiatry should be considered primarily as the manifestations of the modified cognition, emotion and behavior of the patient, leading to a modified inner experience of self, others or the world, and not as the subjective experience of an altered part of the body. This raises important questions about the means to conduct a reliable and valid psychiatric interview (for a review see [50]), about the relationship between semiologic psychiatry and the underlying neurophysiological distal mechanism [2] and about the formation of mental symptoms [47].

As stated by Kendler: “a more comprehensive explanation of a psychiatric disorder must include an understanding of the production of the key symptoms and signs underlying that disorder (...). As we understand the brain processes, we need to "back-translate" the biology into an understanding - in psychological terms - of the keys psychopathological constructs under investigation" [2]. An optimistic and materialist approach could consider that the development of cognitive science and neuroscience in the domain of self-experience and related physiological mechanisms heralds the possibility of organizing symptoms and signs on the basis of physiology. Chalmers called it a "materialism of type C" [56]. This materialism posits that if the laws of current physics do not yet allow for the complete exploration of mental symptoms, there is no a priori explanatory gap concerning the two types of data. The belief is strong that, one day, the development of neurosciences will enable us to account entirely for the whole tasting experience strictly in neurophysiological terms. A less 34 materialist approach, close to the neurophenomenological project $[57,58]$, would involve 
1 describing signs and symptoms on the basis of their qualitative/subjective aspects and evaluating their neurophysiological correlates on the basis of reciprocal constraints, in which qualitative/subjective aspects and neurophysiological data mutually inform each other [57]. "An iterative relationship between psychology and biology, where initial psychological constructs are better defined and subdivided by initial biology findings, which in turn help clarify the biology, will needed to reach a more complete understanding. In short biological and psychological perspectives will coevolve" [2]. The first difficulty of this reciprocal constraint approach is the possibility that symptoms form via different pathways [4, 47].

"Because of the different ways in which internal changes can be interpreted, one biological signal can give rise to different mental symptoms. Similarly, it can be envisaged that different biological signals might be configured as the same mental symptom" [4]. The second difficulty is that it supposes that we need to refine the semiologic register in psychiatry [4]. As Kendler stressed, "empirical research is crucially dependent on the adequacy of the employed phenotypic distinctions, adequacy that cannot be achieved through a simplistic behaviorist checklist approach" [51]. Berrios also clearly highlighted this issue: "mental symptoms became the "atoms" or "units of analysis" of descriptive psychopathology. This language was calibrated according to the epistemological requirements of contemporary clinical practice and research. Importantly, it has changed little since. (...) Consequently, however, this has resulted in a significant mismatch between the resolution power of the language of psychiatry and that of the instruments devised to capture and measure its proxy variables" [4].

In line with the DSM-III position, psychiatric signs and symptoms are primarily mental and a rigorous methodology is required to organize them in psychiatric semiology and to obtain a reliable neuroscientific explanation for them [42, 43, 49, 51]. A challenge in psychiatry is thus to better define and organize a patient's symptoms and signs in terms both of his/her subjacent brain mechanisms and of his/her inner experience of the symptoms and signs (according to a reciprocal constraint approach).

\section{Psychiatric semiology as seen by the causal network model}

The previous latent variable model of semiology posited that symptoms and signs are independent things related together by a latent variable [59]. However, it has also been suggested that symptoms and signs have properties with autonomous causal relevance [60]. "Instead of being effect of common cause, psychiatric symptoms have been argued to cause each other" [59]. Thus, symptoms and signs can be related by causal chains that can be better 
1 studied by a causal network model. This network approach considers symptoms and signs as

2 "nodes" and causal interactions between symptoms as "connections between nodes".

3 According to this point of view, a psychiatric disorder is to be considered as a set of

4 symptoms and signs that are connected thought a system of causal relations [61]. "In such a model, symptoms are not an indicator of an underlying pathological process, rather they are parts of a functionally interconnected causal network" [62].

7 From a clinical point of view, the causal network model helps the psychiatrist in collecting 8 signs and symptoms. Thus, collection is not solely based on the search for a latent 9 physiological mechanism that might explain the manifestation but also on the understanding 10 of the relationship between the symptoms and signs in the experience of the subject $[39,59]$. 11 Indeed, psychiatrists intuitively interpret relations between symptoms as causal networks 12 [63]. For example, the relationship between hallucination, anxiety and insomnia is 13 understandable through their causal relationship without any need for a latent variable. 14 Interestingly, the DSM itself contains criteria with causal relationships, e.g. for the panic 15 disorder: worries about the possible implication of another attack [63], or for the obsessive16 compulsive disorder: compulsion in response to an obsession [59]. However, a limitation of 17 the causal network model is that it is elusive about what symptoms or signs are to be included 18 in the model. Mental symptoms and signs remain the "units of analysis" but their nature, 19 formation and how they are to be captured are not clearly defined [38]. Nevertheless, the semiologic register in psychiatry is not a simple and neutral "description" of reality [4]. It construes mental manifestations in particular ways, a point that has received very little attention in the psychiatric literature on the causal network model. Yet this approach could be of interest to better describe the formation of psychiatric manifestations. Indeed, such a network approach model can include social interactions [64] and their impact on the formation of mental symptoms and signs. As Berrios highlighted, "the final description of symptom results from the interaction between a tenuous biological signal (originated in an affected brain site) and the layers of psychosocial codes (noise) partaking in the process of symptom formation. (...) The crucial point here is that clinical 'observation' is never a cognitively innocent activity" [5]. This view is known as the Cambridge model of symptom formation $[4,47]$, which is related to the hypothesis that the biological/physiological signal (related to a brain modification) is something pre-linguistic and pre-conceptual (also called the "primordial soup"[47]) that needs to be constructed to be communicated according to social configurators (Figure 1) [65]. Further work could investigate how such configurators could be included in the causal network model. 
1 From a physiological point of view, the causal network model is agnostic with regard to how causal relations between symptoms and signs are grounded [59]. However, it involves considering the mechanism underlying the clinical psychiatric manifestation not in terms of a single clear explanation but rather as a multifactorial entity, as with the systemic biological approach to brain functioning [26]. Figure 1. The way in which the psychiatric disorder develops into a set of symptoms and signs that are relatively stable may be related to the process regulating homeostatic brain function. They are more or less stable because they reinforce each other mutually and because such a network may be subsumed by brain homeostatic regulation underpinned by complex and mutually reinforcing networks of causal mechanisms. Figure 1. This implies adding a semiologic level to the traditional multilevel analysis in neuroscience. Interestingly, such a semiologic view would involve identifying not only clearly defined signs and symptoms but also "anti-signs" and "anti-symptoms" that "protect against disorder by blocking the spreading of problems through the network" [60].

The fact that signs and symptoms in psychiatry are neither independent nor passive could be considered as an important difference between psychiatric and non-psychiatric entities. Indeed, the model has also been called the "network approach to psychopathology" [59]. However, the causal network model has also been applied to non-psychiatric disorders, in line with the concept of "diseasome" [66] and "network medicine" [64, 67]. However, in such a model, nodes represent "disorders" and not "symptoms and signs". To our knowledge, the causal network model has not been used for the symptoms and signs of non-psychiatric disorders. Nevertheless, the model has been applied to health-related quality of life in healthy adults and patients with cancer [68], which highlight the interest of such an approach in the domain of chronic diseases to better understand the impact and the relationship of cognition, emotion and behavior on patients' health [69].

The absence of a single latent variable or the absence of a common cause could mean that it is impossible to establish a pragmatic measure of explanation in line with the complexity and the multilevel characteristics of the model. In fact, a "network model load" could be measured and would prove very useful in clinical practice and research. Indeed, multilevel causal chains cutting across levels of organization and timescales can be projected in lower dimensional space. The key challenge is to find the lowest dimensional denominator that explains the behavior of the system and then to extract a metric that is relevant in both nosologic and semiologic terms. Figure 3 provides two examples in which a unidimensional view based on averages does not meaningfully differentiate controls and patients, whereas a 34 multidimensional view allows a problem to be encompassed by a compound metric separating 
1 the two populations. Recent development in machine learning has boosted the use of such

2 multivariate metrics but they still need a clear evaluation of their validity in relation to 3 physiological mechanisms. Evaluation of their reliability is more complex and can even 4 question existing boundaries. The recent development of normative modeling, for instance, 5 makes it possible to move away from a traditional case vs. control approach and to consider 6 more complex scenarios with various spectra and comorbidities [70].

7 A challenge in psychiatry is thus to incorporate the semiologic more meaningfully into a 8 consensual network model that combines social interaction (including with the medical 9 system), psychological processes, neuronal functioning and even genetic data [71]. As we 10 have underlined, the RDoC is based on an integrative, multilevel, multisystem approach. We 11 think that research in semiology should form part of the RDoC project in order to better 12 understand the link between brain dysfunction and the clinical manifestations of disorders 13 [20]. This would link in much better with clinical practice. Nevertheless, two major 14 challenges remaining are: i) to train the new generation of psychiatrists in how such an 15 interdisciplinary perspective may be adopted, and ii) to include digital technology in their 16 training in order to optimize the delivery of psychiatric medicine in the future $[71,72]$. 17 


\section{Conclusion}

2 This article questions the notion that psychiatric disorders are to be considered differently 3 from non-psychiatric disorders. We have shown that debate until now has focused more on 4 the nosologic than on the semiologic. Rather that highlighting specific features of psychiatric 5 disorders, nosologic point of view reveals factors that co-occur across the entire field of 6 medicine if the objective is to establish the boundaries between the normal and the pathologic 7 and between different disorders. Psychiatry is thus very important in medicine because it 8 exemplifies the inherent problem of the construction of a cognitive framework that has been 9 imposed on clinical and scientific medical information to delineate a classification of 10 disorders and to increase its comprehensibility and utility. In this sense, it can be considered 11 that psychiatric disorders are not different from non-psychiatric disorders, provided that care 12 is taken in how non-psychiatric disorders are defined. This paradigm shift is in line with the 13 position of Spitzer and Endicott in 1978 when they stated that "a mental disorder should be 14 defined as merely a subset of medical disorder, and that our attention should therefore be 15 directed toward defining the broad rubric of medical disorder" [21]. Thinking about 16 psychiatric classification should therefore seek to "clarify the goals of medical classification" 17 and offer "a rationale for decisions as to which consideration should be included or excluded 18 from a medical classification" [21].

19 In contrast, semiologic point of view has led to the discovery of psychiatric features that 20 underline the great value of psychiatry and the scientific challenges it faces. This position is 21 in line with Berrios who claims that the "primary objects" of psychiatry are mental symptoms 22 and signs, and that mental disorders are "derivative objects", with higher level organizations 23 [47]. This article has focused on the need to better define and classify signs and symptoms in 24 psychiatry based on a dual, mutually interactive biological and psychological perspective and 25 to incorporate semiologic psychiatry into a multilevel multisystem brain/cognitive approach. 26 In such a view, there is an urgent need to "make semiologic psychiatry great again". As for 27 nosologic issues, an international task force should be set up to investigate semiologic 28 psychiatry in the field of medicine. In this task force, historical, societal, epistemological and 29 phenomenological (in it continental philosophical meaning [10]) aspects concerning mental 30 manifestations discussed in this article should be not forgotten. Moreover, as chronic disease 31 becomes an ever greater burden for society [72], such a task force would have a great effect in 32 better understanding the impact of cognition, emotion and behavior on the onset and 33 maintenance of non-psychiatric disorders beyond the dichotomy between mental and physical 34 disorders. 


\section{Acknowledgements}

2 Many thanks to Ray Cooke for his very careful English editing.

3

4

\section{Declaration of interest}

6 The authors declare no potential conflicts of interest with respect to the research, authorship 7 and/or publication of this article.

8

9

10 Funding

11 The authors received no financial support for the research, authorship and/or publication of 12 this article. 
3 [1] Kendler KS, Zachar P, Craver C. What kinds of things are psychiatric disorders? 4 Psychol Med 2011;41:1143-50.

5 [2] Kendler KS. Explanatory models for psychiatric illness. Am J Psychiatry 6 2008;165:695-702.

7 [3] Aragona M. The concept of mental disorder and the DSM-V. Dial Phil Ment Neuro Sci $8 \quad 2009 ; 21-14$

9 [4] Markova IS, Berrios GE. Epistemology of psychiatry. Psychopathology 2012;45:220107.

11 [5] Berrios G. The History of Mental Symptoms: Descriptive Psychopathology since the 12 Nineteenth Century. Cambridge: Cambridge University Press 1996.

13 [6] Grinker RR, Sr. The sciences of psychiatry: fields, fences and riders. Am J Psychiatry 14 1965;122:367-76.

15 [7] Clark LA, Cuthbert B, Lewis-Fernandez R, et al. Three Approaches to Understanding 16 and Classifying Mental Disorder: ICD-11, DSM-5, and the National Institute of Mental 17 Health's Research Domain Criteria (RDoC). Psychol Sci Public Interest 2017;18:72-145.

18 [8] Amad A, Fovet T, Geoffroy PA. Keep calm: Psychiatric disorders are organic! The 19 power of words in medicine. Aust N Z J Psychiatry 2015;50:100-1.

20 [9] Micoulaud-Franchi JA, Quiles C, Masson M. Keep calm and carry on: Mental disorder 21 is not more "organic" than any other medical condition. Encephale 2017.

22 [10] Berrios GE. What is phenomenology? A review. J R Soc Med 1989;82:425-8.

23 [11] Beumont PJ. Phenomenology and the history of psychiatry. Aust N Z J Psychiatry 24 1992;26:532-45.

25 [12] Aragona M. Rethinking received views on the history of psychiatric nosology: Minor 26 shifts, major continuities. In: Zachar P, Stoyanov D, Aragona M, et al., editors. Alternative 27 perspectives on psychiatric validation DSM, ICD, RDoC, and beyond. Oxford: Oxford 28 University Press; 2015. p. 27-46.

29 [13] American Psychiatric Association. Diagnostic and Statistical Manual of Mental 30 Disorder, 5th ed, Text Revision (DSM-5). Washington, DC: American Psychiatric 31 Association; 2013.

32 [14] Spitzer RL, Endicott J, Robins E. Research diagnostic criteria: rationale and 33 reliability. Arch Gen Psychiatry 1978;35:773-82. 
1 [15] Feighner JP, Robins E, Guze SB, et al. Diagnostic criteria for use in psychiatric 2 research. Arch Gen Psychiatry 1972;26:57-63.

3 [16] Wilson M. DSM-III and the transformation of American psychiatry: a history. Am J 4 Psychiatry 1993;150:399-410.

5 [17] American Psychiatric Association. Diagnostic and Statistical Manual of Mental 6 Disorder, 3th ed, (DSM-III). Washington, DC: American Psychiatric Association; 1980.

7 [18] Cuthbert BN. The RDoC framework: facilitating transition from ICD/DSM to 8 dimensional approaches that integrate neuroscience and psychopathology. World 9 Psychiatry 2014;13:28-35.

10 [19] Kapur S, Phillips AG, Insel TR. Why has it taken so long for biological psychiatry to 11 develop clinical tests and what to do about it? Mol Psychiatry 2012;17:1174-9.

12 [20] Cuthbert BN, Insel TR. Toward the future of psychiatric diagnosis: the seven pillars 13 of RDoC. BMC Med 2013;11:126.

14 [21] Spitzer R, Endicott J. Medical and mental disorder: proposed definition and criteria. 15 In: Spitzer R, Klein D, editors. Critical issues in psychiatric diagnosis. New York: Raven 16 Press; 1978.

17 [22] Weinberger DR, Goldberg TE. RDoCs redux. World Psychiatry 2014;13:36-8.

18 [23] McGorry P, Keshavan M, Goldstone S, et al. Biomarkers and clinical staging in 19 psychiatry. World Psychiatry 2014;13:211-23.

20 [24] Loth E, Spooren W, Ham LM, et al. Identification and validation of biomarkers for 21 autism spectrum disorders. Nat Rev Drug Discov 2016;15:70-3.

22 [25] Chen R, Shi L, Hakenberg J, et al. Analysis of 589,306 genomes identifies individuals 23 resilient to severe Mendelian childhood diseases. Nat Biotechnol 2016;34:531-8.

24 [26] Kendler KS. Levels of explanation in psychiatric and substance use disorders: 25 implications for the development of an etiologically based nosology. Mol Psychiatry 26 2012;17:11-21.

27 [27] Micoulaud Franchi JA, Dumas G, Quiles C, et al. [From clinic to the "foul and exciting 28 field of life": A psychiatric point of view on clinical physiology]. Annales Medico 29 Psychologiques 2017;175:70-85.

30 [28] Fava GA, Rafanelli C, Tomba E. The clinical process in psychiatry: a clinimetric 31 approach. J Clin Psychiatry 2012;73:177-84.

32 [29] Fava GA. Road to nowhere. World Psychiatry 2014;13:49-50. 
1 [30] Robins E, Guze SB. Establishment of diagnostic validity in psychiatric illness: its 2 application to schizophrenia. Am J Psychiatry 1970;126:983-7.

3 [31] Kendler KS. Toward a scientific psychiatric nosology. Strengths and limitations. 4 Arch Gen Psychiatry 1990;47:969-73.

5 [32] Keshavan MS, Ongur D. The journey from RDC/DSM diagnoses toward RDoC 6 dimensions. World Psychiatry 2014;13:44-6.

7 [33] Stein DJ. An integrative approach to psychiatric diagnosis and research. World 8 Psychiatry 2014;13:51-3.

9 [34] Kendler KS. Toward a limited realism for psychiatric nosology based on the 10 coherence theory of truth. Psychol Med 2015;45:1115-8.

11 [35] Belzung C, Billette de Villemeur E, Lemoine $\mathrm{M}$, et al. Latent variables and the 12 network perspective. Behav Brain Sci 2010;33:150-1.

13 [36] Phillips MR. Will RDoC hasten the decline of America's global leadership role in 14 mental health? World Psychiatry 2014;13:40-1.

15 [37] Frances A. RDoC is necessary, but very oversold. World Psychiatry 2014;13:47-9.

16 [38] Markova IS, Berrios GE. Epistemology of mental symptoms. Psychopathology $17 \quad 2009 ; 42: 343-9$.

18 [39] Nordgaard J, Sass LA, Parnas J. The psychiatric interview: validity, structure, and 19 subjectivity. Eur Arch Psychiatry Clin Neurosci 2013;263:353-64.

20 [40] Wing JK, Cooper JE, Sartorius N. The measurement and classification of psychiatric 21 symptoms. Cambridge: Cambridge University Press; 1974.

22 [41] Spitzer RL. Psychiatric diagnosis: are clinicians still necessary? Compr Psychiatry 23 1983;24:399-411.

24 [42] Andreasen NC. DSM and the death of phenomenology in America: an example of 25 unintended consequences. Schizophr Bull 2007;33:108-12.

26 [43] Parnas J. The Breivik case and "conditio psychiatrica". World Psychiatry $27 \quad 2013 ; 12: 22-3$.

28 [44] Wakefield JC. Wittgenstein's nightmare: why the RDoC grid needs a conceptual 29 dimension. World Psychiatry 2014;13:38-40.

30 [45] Fellowes S. Symptom modelling can be influenced by psychiatric categories: choices 31 for research domain criteria (RDoC). Theor Med Bioeth 2017;38:279-94.

32 [46] Berrios GE, Chen EY. Recognising psychiatric symptoms. Relevance to the 33 diagnostic process. Br J Psychiatry 1993;163:308-14. 
1 [47] Berrios G. Formation and meaning of mental symptoms: history and epistemology.

2 Dial Phil Ment Neuro Sci 2013;6:39-48.

3 [48] Maj M. Keeping an open attitude towards the RDoC project. World Psychiatry $4 \quad 2014 ; 13: 1-3$.

5 [49] Parnas J, Sass LA, Zahavi D. Rediscovering psychopathology: the epistemology and 6 phenomenology of the psychiatric object. Schizophr Bull 2012;39:270-7.

7 [50] Nordgaard J, Revsbech R, Saebye D, et al. Assessing the diagnostic validity of a 8 structured psychiatric interview in a first-admission hospital sample. World Psychiatry 9 2012;11:181-5.

10 [51] Parnas J. The RDoC program: psychiatry without psyche? World Psychiatry $112014 ; 13: 46-7$.

12 [52] Luders H, Acharya J, Baumgartner C, et al. Semiological seizure classification. 13 Epilepsia 1998;39:1006-13.

14 [53] Engel J, Jr. A proposed diagnostic scheme for people with epileptic seizures and 15 with epilepsy: report of the ILAE Task Force on Classification and Terminology. 16 Epilepsia 2001;42:796-803.

17 [54] Donen S, Kelly G. Singing in the brain. Los Angeles: MGM Inc Press; 1956.

18 [55] Micoulaud Franchi JA, Lancon C. L'inaccessible presque touché. Connaissance 19 minimale en psychologie de la santé à l'usage de l'étudiant en médecine. Annales Medico 20 Psychologiques 2015;173:377-83.

21 [56] Chalmers D. The character of consciousness. Oxford/New-York: Oxford university 22 Press; 2010.

23 [57] Varela F. Neurophenomenology: A methodological remedy for the hard problem. . 24 Journal of Consciousness Studies 1996;3:330-35.

25 [58] Gallagher S, Brosted Sorensen J. Experimenting with phenomenology. Conscious 26 Cogn 2006;15:119-34.

27 [59] Borsboom D. A network theory of mental disorders. World Psychiatry 2017;16:52813

29 [60] Borsboom D, Cramer AO, Schmittmann VD, et al. The small world of 30 psychopathology. PLoS One 2011;6:e27407.

31 [61] Borsboom D. Psychometric perspectives on diagnostic systems. J Clin Psychol 32 2008;64:1089-108. 
1 [62] Zachar P, Kendler KS. The Philosophy of Nosology. Annu Rev Clin Psychol 2 2017;13:49-71.

3 [63] Nuijten M, Deserno M, Cramer A, et al. Mental disorders as complex networks: An 4 introduction and overview of a network approach to psychopathology Clin 5 Neuropsychiatry 2016;13:68-76.

6 [64] Barabasi AL. Network medicine--from obesity to the "diseasome". N Engl J Med $7 \quad 2007 ; 357: 404-7$.

8 [65] Berrios GE. Descriptive psychopathology: conceptual and historical aspects. Psychol 9 Med 1984;14:303-13.

10 [66] Goh KI, Cusick ME, Valle D, et al. The human disease network. Proc Natl Acad Sci U S 11 A 2007;104:8685-90.

12 [67] Barabasi AL, Gulbahce N, Loscalzo J. Network medicine: a network-based approach 13 to human disease. Nat Rev Genet 2011;12:56-68.

14 [68] Kossakowski JJ, Epskamp S, Kieffer JM, et al. The application of a network approach 15 to Health-Related Quality of Life (HRQoL): introducing a new method for assessing 16 HRQoL in healthy adults and cancer patients. Qual Life Res 2016;25:781-92.

17 [69] Bragazzi NL. Rethinking psychiatry with OMICS science in the age of personalized 18 P5 medicine: ready for psychiatome? Philos Ethics Humanit Med 2013;8:4.

19 [70] Marquand AF, Rezek I, Buitelaar J, et al. Understanding Heterogeneity in Clinical 20 Cohorts Using Normative Models: Beyond Case-Control Studies. Biol Psychiatry $212016 ; 80: 552-61$.

22 [71] Bhugra D, Tasman A, Pathare S, et al. The WPA-Lancet Psychiatry Commission on 23 the Future of Psychiatry. Lancet Psychiatry 2017;4:775-818.

24 [72] Insel TR. Digital Phenotyping: Technology for a New Science of Behavior. JAMA 25 2017;318:1215-6. 
1 Table 1: The Spitzer \& Endicott 1978 criteria regarding the boundaries between the normal 2 and the pathologic [14].

3

All four criteria, A through $\mathrm{D}$, must be met for a condition to be designated as a medical disorder.

Criterion A. The condition, in the fully developed or extreme form, in all environments (other than one especially created to compensate for the condition), is directly associated with at least one of the following:

1. distress - acknowledged by the individual or manifested;

2. disability—some impairment in functioning in a wide range of activities;

3. disadvantage (not resulting from the above) — certain forms of disadvantage to the individual in interacting with aspects of the physical or social environment because of an identifiable psychological or physical factor. The following forms of disadvantage, even when not associated with distress or disability, are now considered, in our culture, as suggestive of some type of organismic dysfunction warranting the designation of medical disorder:

a. impaired ability to make important environmental discriminations (e.g., color blindness, lack of pain perception).

b. lack of ability to reproduce (e.g., sterility).

c. cosmetically unattractive because of a deviation in kind, rather

than degree, from physical structure (e.g., fused toes).

d. atypical and inflexible sexual or other impulse-driven behavior which often leads to painful consequences (e.g., sexual sadism, kleptomania, pathological gambling).

e. impairment in the ability to experience sexual pleasure in an interpersonal context (e.g., fetishism).

f. marked impairment in the ability to form relatively lasting and non-conflictual interpersonal relationships (e.g., narcissistic and hysterical personality disorders).

Criterion B. The controlling variables tend to be attributed to being largely within the organism with regard to either initiating or maintaining the condition. Operationally, this criterion is determined by the following: A condition is included [as a disorder] only if it meets both of the following criteria:

1. Simple informative or standard educational procedures do not lead to a reversal of the condition.

\section{Nontechnical interventions do not bring about a quick reversal of the condition.}

Criterion $\boldsymbol{C}$. Conditions are not included if the associated distress, disability, or other disadvantage is apparently the necessary price associated with attaining some positive goal.

Criterion D. Distinctness from other conditions in one or more of the following features: clinical phenomenology, course, response to treatment, familial incidence, or etiology. 
1 Table 2: Operational criteria for setting the boundaries between the normal and the 2 pathologic in the DSM III, IV and 5.

3

\begin{tabular}{|l}
\hline DSM "Inclusion criteria" to identify signs and "Exclusion criteria" to identify signs and \\
\\
symptoms that will be considered as symptoms that will not be considered as \\
"clinically significant" \\
"chathologic
\end{tabular}

DSM- "In DSM-III each of the mental disorders is
III
conceptualized as a clinically significant
behavioral or psychological syndrome or
pattern that occurs in an individual and that is
typically associated with either a painful
symptom (distress) or impairment in one or
more important areas of functioning
(disability)".

DSM- "In DSM-IV, each of the mental disorders is "In addition, there is an inference that there is a behavioral, psychological, or biological dysfunction, and that the disturbance is not only in the relationship between the individual and society. (When the disturbance is limited to a conflict between an individual and society, this may represent social deviance, which may or may not be commendable, but is not by itself a mental disorder.)".

IV conceptualized as a clinically significant "In addition, this syndrome or pattern must not be behavioral or psychological syndrome or pattern that occurs in an individual and that is associated with present distress (e.g., a painful symptom) or disability (i.e., impairment in one or more important areas of functioning) or with a significantly increased risk of suffering death, pain, disability, or an important loss of freedom"

merely an expectable and culturally sanctioned response to a particular event, for example, the death of a loved one. Whatever its original cause, it must currently be considered a manifestation of a behavioral, psychological, or biological dysfunction in the individual. Neither deviant behavior (e.g., political, religious, or sexual) nor conflicts that are primarily between the individual and society are mental disorders unless the deviance or conflict is a symptom of a dysfunction in the individual, as described above."

DSM-5 "A mental disorder is a syndrome characterized The signs and symptoms should "reflect a by clinically significant disturbance in an dysfunction in the psychological, biological, or individual's cognition, emotion regulation, or behavior (...). Mental disorders are usually associated with significant distress or disability in social, occupational, or other important activities".

developmental processes underlying mental functioning. (...) An expectable or culturally approved response to a common stressor or loss, such as the death of a loved one, is not a mental disorder. Socially deviant behavior (e.g., political, religious, or sexual) and conflicts that are primarily between the individual and society are not mental disorders unless the deviance or conflict results from a dysfunction in the individual, as described above." 
1 Table 3: Validity criteria to classify different disorders

2

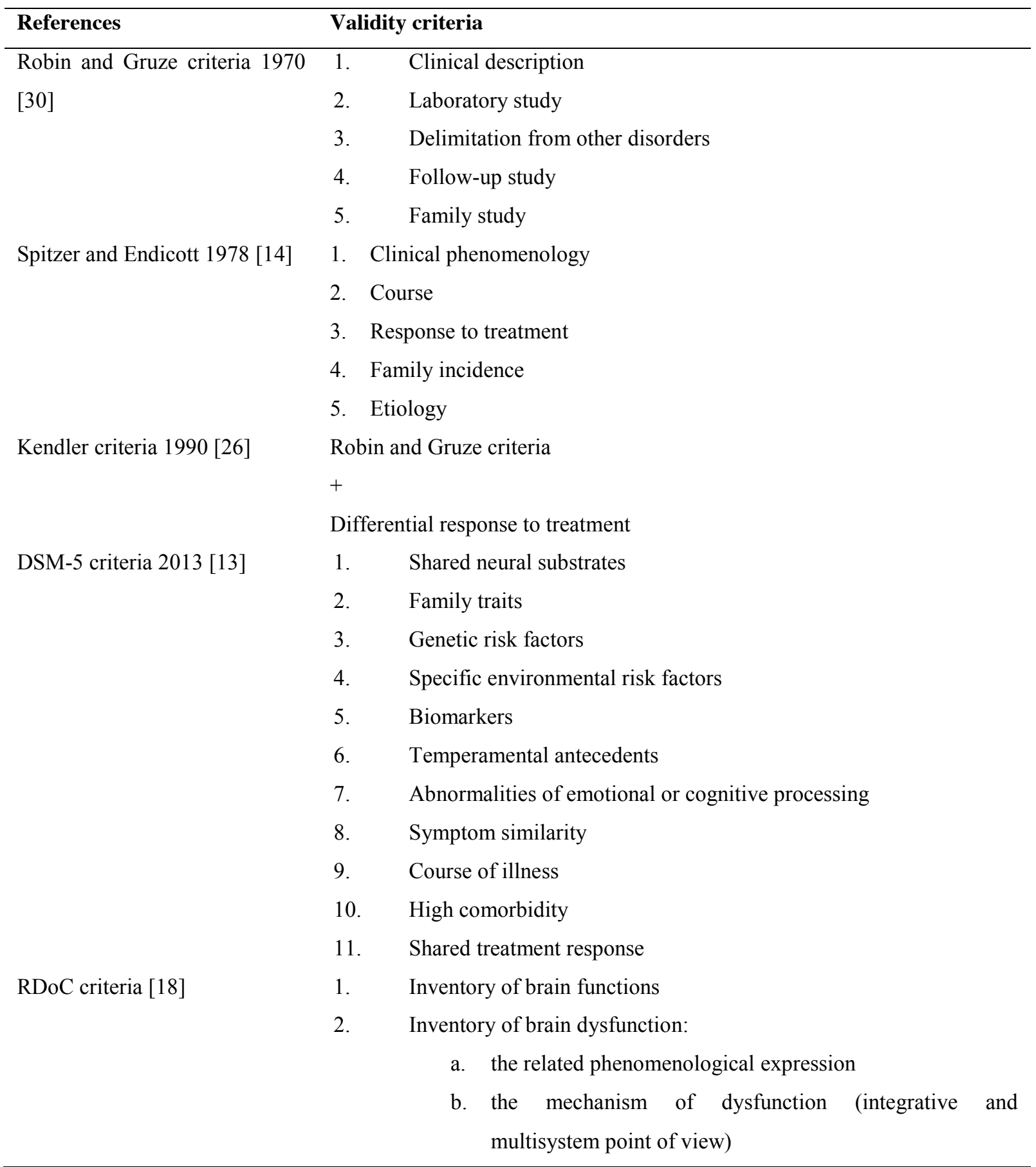




\section{$1 \quad$ Figure 1}

2 Proposition for framework to better identify nosologic and semiologic issues in psychiatry.

3 Left part of the figure: delimiting psychiatric disorders (nosologic level) is not only based on

4 brain constraints that reflect pathophysiological mechanisms, but also on the semiologic level

5 and on epidemiological, prognostic, therapeutic and economic factors [27].

6 Right part of the figure: adaptation of the Cambridge model of symptom formation [47],

7 which posits that mental manifestations, i.e. the semiologic level, result from interactions

8 between brain constraints and social constraints, which in turn are related to the formation and

9 expression of symptoms and signs within a social network.

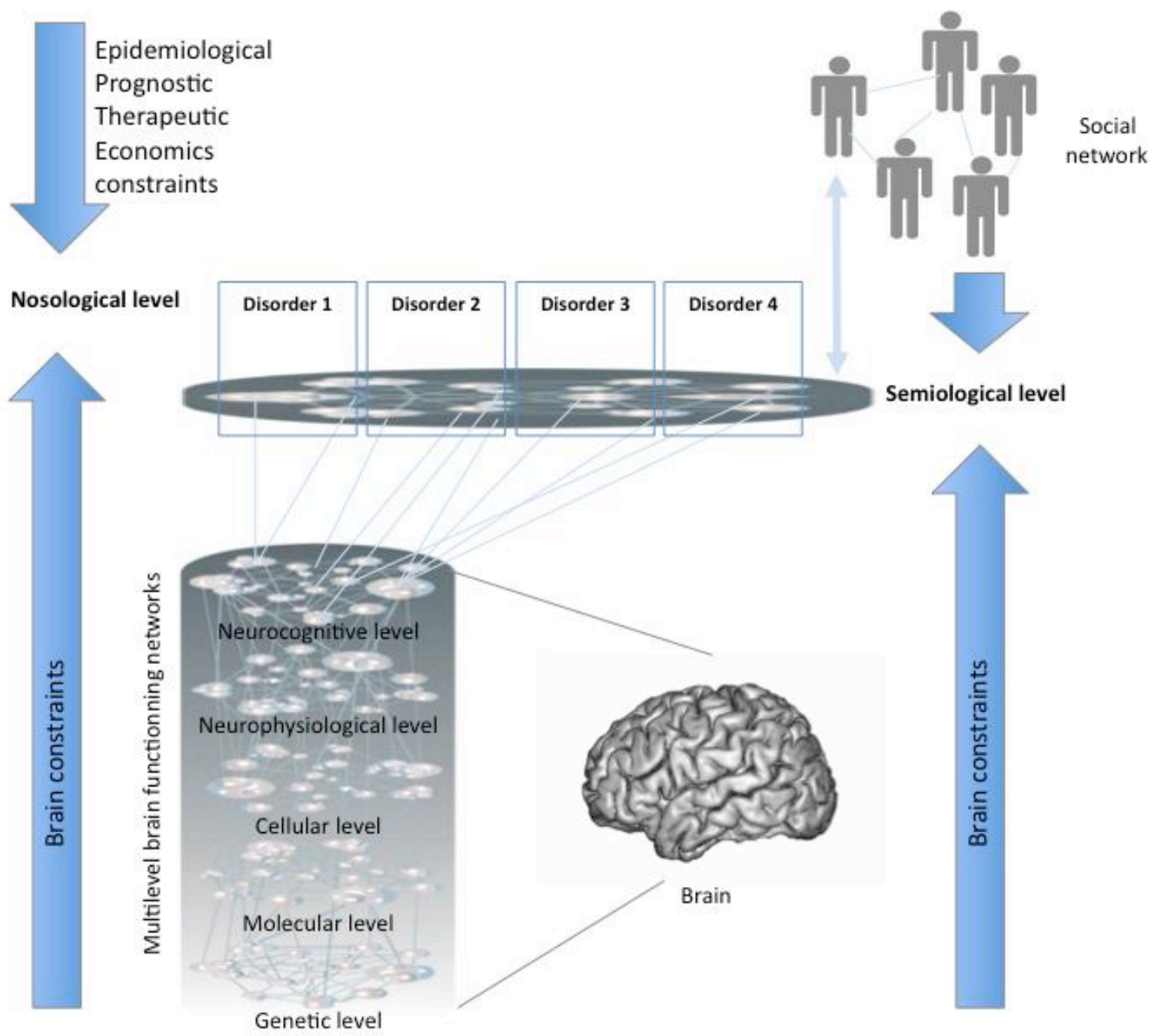




\section{Figure 2}

2 Relation between semiologic psychiatry, RDC/DSM project and RDoC project [12].

3

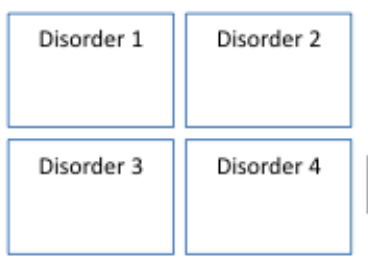

Impact of disorder classification on clinical description

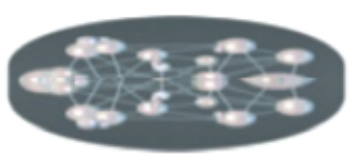

Redefining psychaitric semiological classification

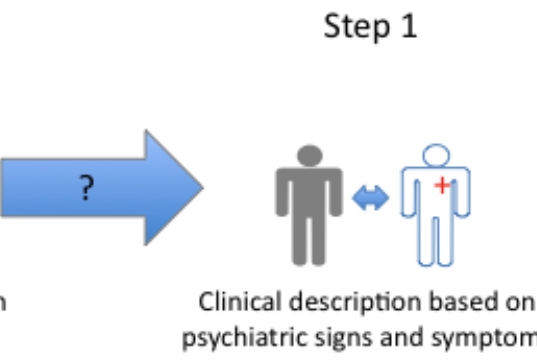

psychiatric signs and symptoms

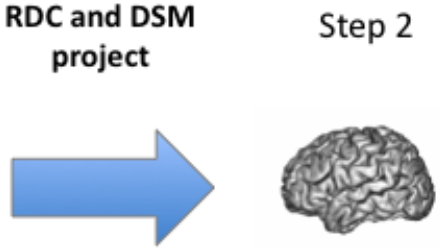

Disorder theory based on neurophysiological mechanism
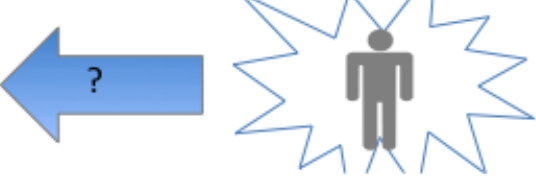

Clinical manifestation Dimensional approach

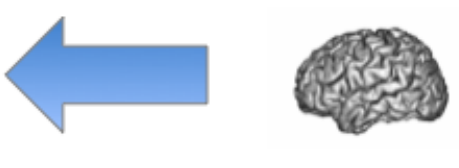

Brain functions and mechanism of dysfunction

$\begin{array}{lcc}\text { Step } 2 & \text { RDoC } & \text { Step } 1\end{array}$




\section{Figure 3}

2 Sometimes, one need to use multi-dimensional spaces to be able to find a simpler

3 unidimensional metric. A, B) two common cases where two variables can be simplified into

4 one metric: A) interaction between cognitive scores 1 and 2 and B) offset in the cognitive

5 score 1 compared to cognitive score 2. Difference between groups remains hidden when

6 averaging across the age dimension; C) example of a more complex landscape with helicoidal

7 trajectories in three dimensions. The two brain measures can be reduced to a unidimensional

8 composite score by calculating the distance $\mathrm{R}$ between each participant and the axis A. D)

9 The new composite score better explains the separation between the groups.

A
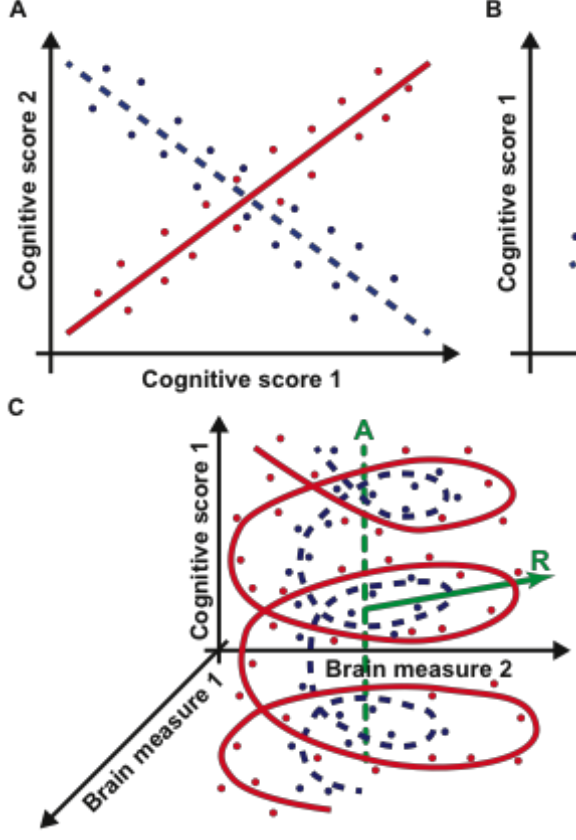

B
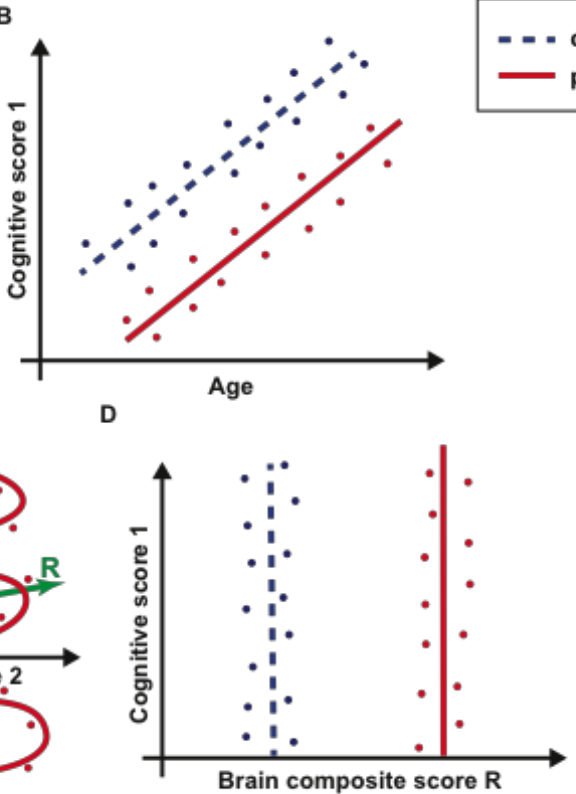

11

12 